\title{
Terapias espirituais e complementares no tratamento do câncer: a experiência de pacientes oncológicos em Florianópolis (SC)
}

\author{
Spiritual and complementary therapies to treat cancer: the experience \\ of oncologic patients in Florianópolis, Santa Catarina, Brazil
}

\author{
Waleska de Araújo Aureliano ${ }^{1}$
}

\begin{abstract}
Resumo
A pesquisa investigou o uso de terapias complementares e espirituais por pacientes oncológicos atendidos em uma instituição terapêutico-religiosa da cidade de Florianópolis (SC), o Centro de Apoio ao Paciente com Câncer (CAPC). Esta instituição oferece terapias complementares, grupos psicoterapêuticos e cirurgias espirituais, sendo que nela trabalham médicos, enfermeiros, religiosos e terapeutas diversos. Utilizamos como método a observação participante realizada ao longo de 15 meses dentro da instituição. Foram realizadas ainda 21 entrevistas semiestruturadas com pacientes e voluntários do CAPC e com seus dirigentes. Observou-se que as pessoas com câncer atendidas no CAPC possuem uma lógica de cuidado diversificada e plural que não opera com as dicotomias mente/corpo, biomedicina/terapias complementares para pensar os processos de saúde e doença, de modo que as terapias complementares/espirituais são analisadas enquanto sistemas possíveis de tratamento. Foram questionadas certas concepções ainda vigentes que analisam a adesão aos tratamentos complementares/espirituais com base nas ideias de "crença", falta de acesso ao sistema biomédico, carência econômica e/ou incapacidade da biomedicina em tratar certas doenças, pois identificou-se na pesquisa que tais formas terapêuticas eram utilizadas por pessoas de diferentes classes sociais e filiações religiosas, em diversos estágios do câncer, paralelamente ao tratamento biomédico, e não como a "última opção".
\end{abstract}

Palavras-chave: câncer; terapias complementares; terapias espirituais.

\begin{abstract}
The objective of this research was to investigate the use of complementary and spiritual therapies for cancer patients treated in a therapeutic-religious institution in the city of Florianópolis, the Center for Cancer Patient Support (CAPC). This institution offers complementary therapies, psychotherapeutic groups and "spiritual surgeries". Doctors, nurses, therapists and religious people work together in this institution. The participant observation method was used during fieldwork conducted over 15 months in the institution. Also, 21 semi-structured interviews with patients and volunteers from CAPC and its leaders were conducted. People being treated for cancer with the complementary/spiritual therapies offered by CAPC presented a diverse and plural understanding of care that does not work with the dichotomies of mind/body, biomedicine/complementary therapies to consider their processes of health and disease. Prevailing concepts that examine adherence to complementary/spiritual therapies based on the ideas of "belief," lack of access to official medical system, economic deprivation and/or inability of biomedicine to treat certain diseases were questioned because it was identified in research that such forms of therapy were used by people of different social classes and religious affiliations, in various stages of cancer, together with the biomedical treatment, and not as the "last option".
\end{abstract}

Keywords: cancer; complementary therapies; spiritual therapies.

'Doutora em Antropologia Social pela Universidade Federal de Santa Catarina (UFSC) - Florianópolis (SC), Brasil. Endereço para correspondência: Waleska de Araújo Aureliano - Rua São Francisco Xavier, 478/1203 - Maracanã - CEP: 20550-013 - Rio de Janeiro (RJ), Brasil - E-mail: waureliano26@yahoo.com.br

Conflito de interesse: nada a declarar. 


\section{INTRODUÇÃO}

Neste artigo, foram apresentados dados de pesquisa realizada junto a um centro de tratamento para pacientes oncológicos localizado em Florianópolis (SC), o Centro de Apoio ao Paciente com Câncer (CAPC). Este centro oferece terapias complementares, grupos psicoterapêuticos e cirurgias espirituais como formas terapêuticas de apoio ao tratamento do câncer.

A pesquisa teve por objetivo investigar como o tratamento complementar/espiritual se inseria no itinerário terapêutico do paciente oncológico e como os pacientes relacionavam as terapias convencionais para o câncer e aquelas recebidas neste centro. Questionou-se ainda em que medida as questões relacionadas à classe social, acesso a sistemas médicos, gravidade da doença e pertencimento religioso influenciavam a escolha por esse tipo de tratamento.

Apesar do uso crescente da medicina alternativa/complementar (MAC), do seu reconhecimento por organismos oficiais de saúde ${ }^{1}$ e da introdução de práticas complementares e integrativas em sistemas públicos de saúde ${ }^{2}$, ainda há poucos estudos que tematizem a percepção dos pacientes sobre o uso dessas formas terapêuticas.

Segundo Spadacio e Barros ${ }^{3}$, a maioria das pesquisas sobre MAC ainda é de cunho quantitativo e concentrada no hemisfério norte. $\mathrm{O}$ estudo realizado pelos autores ${ }^{3}$ baseou-se na análise de 43 artigos científicos que abordaram a utilização de MAC por pacientes oncológicos. A conclusão foi de que essas formas de tratamento fariam parte do escopo social dos pacientes, porém, os autores não apresentaram dados qualitativos e de pesquisa de campo, já que a proposta de sua investigação estava numa revisão sistemática da literatura médica.

Luz $^{4}$ faz uma análise macrossocial do crescimento do uso de MAC no contemporâneo, apontando alguns fatores que podem explicar a expansão e a legitimação gradual dessas práticas na atualidade, entre eles: o encontro cultural das medicinas tradicionais com novas concepções sobre saúde, adoecimento, cura e as relações homem/natureza presentes na sociedade civil; o fato de a MAC oferecer um modelo diferente da relação médico/paciente, que atrai tanto terapeutas quanto pacientes; o crescimento dentro da própria biomedicina de concepções e teorias psicossociais para pensar o adoecimento visando superar a dicotomia mente/corpo da cultura ocidental; e, principalmente, a crise da saúde e da medicina, que obrigou o reconhecimento das limitações da prática biomédica. No entanto, o estudo 4 não aborda aspectos socioculturais em micronível de análise relativo aos sujeitos específicos que se utilizam ou oferecem tratamentos por meio da MAC como médicos, terapeutas, religiosos e pacientes.

Já Barros ${ }^{5}$ analisa o campo da MAC a partir dos profissionais biomédicos, classificando como: ortodoxos os que não aceitam outras formas de cuidado além da biomedicina; alternativos, ou seja, aqueles que foram formados dentro da racionalidade médica científica, porém romperam completamente com ela para atuar apenas no campo da MAC; e, por fim, os híbridos, que embora semelhantes aos demais pela formação biomédica, diferem-se por conciliarem diferentes práticas médicas e por buscarem espaços legítimos e oficiais para promover essa integração. $\mathrm{O}$ autor ${ }^{5}$, no entanto, não aborda a perspectiva dos pacientes e como estes relacionam as práticas de cura da MAC com aquelas advindas da biomedicina.

A partir de estudo de caso baseado em abordagem socioantropológica, procurou-se suprimir de certa forma essa lacuna ao se analisar a percepção de pacientes oncológicos sobre o uso de MAC e terapias espirituais no tratamento do câncer. $\mathrm{Na}$ antropologia, há uma literatura considerável que trata das concepções de saúde e formas de tratamento diversas da biomedicina, impossível de ser revisada no escopo de um artigo. Pode-se dizer que a antropologia da saúde nasce da observação dos primeiros antropólogos sobre as sociedades tribais ${ }^{6}$, nas quais as questões de saúde encontravam-se imbricadas com os demais campos da vida social, especialmente a religião.

No Brasil, estudos antropológicos têm investigado diversas práticas de cura e concepções de saúde presentes em diferentes contextos: religioso ${ }^{7-12}$, nos grupos indígenas ${ }^{13,14} \mathrm{e}$, mais recentemente, nas formas terapêuticas alternativas surgidas com o advento da Nova Era, e utilizadas por segmentos das classes médias urbanas ${ }^{15-18}$.

No entanto, ainda são escassas as investigações que tratam especificamente do uso de terapias complementares/alternativas entre pacientes com câncer. Podemos citar uma pesquisa ${ }^{19}$ que investigou o uso da MAC em pacientes oncológicos na Turquia, e outra investigação semelhante na Argentina ${ }^{20}$. Também cabe mencionar a publicação francesa Anthropologie \& Santé, que em 2011 trouxe um número especial com resultados de pesquisas sobre tratamentos não convencionais para o câncer ${ }^{21}$.

Esta pesquisa não teve a intenção de testar a eficácia das terapias complementares e espirituais oferecidas pelo CAPC, mas analisar quais significados adquirem para pacientes oncológicos e como são articuladas (ou não) aos tratamentos convencionais para o câncer.

\section{METODOLOGIA}

A pesquisa, de caráter qualitativo, desenvolveu-se principalmente por meio da observação participante realizada no CAPC. A pesquisa de campo durou ao todo 15 meses, entre os anos de 2009 e 2010, durante os quais foi acompanhada a rotina da instituição, com acesso direto às práticas terapêuticas ali realizadas. A observação participante foi desenvolvida adaptando-se o 
método antropológico clássico, originalmente desenvolvido para análise das sociedades tribais, para as sociedades urbanas modernas $^{22}$.

Além da observação participante, foram realizadas 21 entrevistas semiestruturadas com pacientes e voluntários (ex-pacientes) do CAPC. Para as entrevistas, buscou-se selecionar pessoas de diferentes idades, filiação religiosa, formação profissional, estágio da doença e classe social a fim de analisar de que forma essas variáveis poderiam estar relacionadas à busca do tratamento complementar/espiritual. Foram selecionados 7 homens e 14 mulheres, refletindo a maior proporção de mulheres como pacientes do CAPC. A faixa etária variou entre 27 e 60 anos, sendo a média de 53 anos.

Para análise dos dados qualitativos, a base foram a análise de narrativas ${ }^{23-26}$ e o paradigma da performance ${ }^{27-29}$. Na antropologia da saúde, as narrativas têm sido analisadas como meio primário para objetificar e dar forma à experiência da doença, tornando-a disponível para o próprio sujeito que fala ${ }^{23}$. Narrar a experiência individual com a doença possibilitaria à pessoa doente colocar-se não apenas diante do outro, mas também diante de si mesma, dando coerência e conteúdo à sua história ${ }^{24}$. Por outro lado, as narrativas devem ser observadas enquanto um aspecto integral da interação entre as pessoas, que faz parte do processo de construção social da realidade, não sendo apenas relatos a posteriori de eventos vividos e distanciados da ação social e da prática ${ }^{25,26}$.

Aliado à análise das narrativas utilizou-se o paradigma da performance como ferramenta analítica. A performance pode ser definida como um evento que coloca a experiência dos sujeitos em relevo, produzindo um estranhamento do cotidiano que os conduz à reflexividade ${ }^{28}$. Este evento está situado num contexto particular e temporário, e visa oferecer aos sujeitos experiências cujo objetivo seria a transformação que rejeita qualquer separação entre o racional, o emocional e o corporal. A participação no tratamento complementar/espiritual do CAPC foi analisada como um evento de performance, pois ficou claro que essa experiência significava, para a maioria dos pacientes, um momento importante, no qual eles podiam voltar-se integralmente para sua experiência com o câncer e refletir sobre seu processo de cura.

A pesquisa seguiu as exigências da Resolução 196/1996 ${ }^{30}$, incluindo o consentimento informado das pessoas entrevistadas, e o projeto de pesquisa foi aprovado pelo Comitê de Ética em Pesquisa com Seres Humanos da Universidade Federal de Santa Catarina (UFSC).

\section{O Lócus da Pesquisa: Centro de Apoio ao Paciente com Câncer (CAPC)}

Antes de apresentar os resultados da pesquisa, o CAPC será descrito para que o leitor receba informações gerais sobre o funcionamento dessa instituição, os sujeitos que dela fazem partem e as práticas que ali se desenvolvem.

O CAPC foi fundado em março de 1998, no bairro do Ribeirão da Ilha, na área insular de Florianópolis, e está vinculado ao Núcleo Espírita Nosso Lar (NENL), localizado na cidade de São José, a apenas nove quilômetros de Florianópolis. Na época desta pesquisa, o CAPC atendia em média 100 pacientes por semana, oriundos não apenas de Florianópolis, mas de outras cidades catarinenses, dos estados vizinhos e, eventualmente, de outros países.

Para iniciar um tratamento no CAPC, o paciente com câncer deve passar por atendimento prévio no NENL, onde seus exames clínicos são analisados. O CAPC não realiza atendimento sem a apresentação desses exames, que vão nortear as aplicações terapêuticas e as cirurgias espirituais e comprovar que a pessoa está sendo acompanhada por médicos. $\mathrm{O}$ tratamento inicia-se às terças-feiras e termina aos sábados. Durante a semana, os pacientes ficam internados no regime hospital-dia: recebem as terapias ao longo do dia e retornam para casa no início da noite. O CAPC oferece cerca de 20 terapias complementares, algumas bastante conhecidas, como: os Florais de Bach, a Cromoterapia, o Reiki, a Hidroterapia e fitoterápicos em geral, além das terapias espíritas tradicionais, como os passes e a água fluidificada. Apenas às sextas-feiras os pacientes pernoitam no CAPC para serem submetidos à cirurgia espiritual, que é realizada sem cortes ou qualquer procedimento invasivo.

No CAPC trabalham vários profissionais de saúde, terapeutas diversos e pessoas leigas, e estes são treinados para a aplicação das terapias. Os profissionais da limpeza, da cozinha e a equipe de enfermagem são remunerados, porém, todos os demais trabalhadores são voluntários, inclusive os médicos. Estes atuam tanto dentro da sua profissão, realizando análise de exames e atendimento médico aos pacientes, quanto na aplicação das terapias complementares, sendo alguns deles os médiuns que realizam as cirurgias espirituais.

Apesar de ser uma instituição ligada ao espiritismo, não há impedimento para que pessoas de outras religiões trabalhem como voluntárias no CAPC, que é apresentado como espaço terapêutico, e não de doutrinação espírita. Assim, há voluntários que se definem como católicos, umbandistas, budistas, ou sem religião específica, denominando-se "espiritualistas".

O CAPC assemelha-se a um hospital ou clínica. Uma forte conexão com símbolos biomédicos pode ser observada na organização do espaço físico (enfermaria com 57 leitos, salas de cirurgia, sala de espera e auditório), no modo de vestir dos voluntários (roupa branca, jaleco, máscaras e touca), na hierarquização das funções (médiuns operadores, médiuns doadores, instrumentadores cirúrgicos, auxiliares técnicos, leitor/a de 
prontuários) e na forma como as cirurgias espirituais são realizadas (em macas, com uso de iodo, gazes, bisturis de fio cego, curativos, emissão de atestados e orientações pós-cirúrgicas). O centro dispõe ainda de uma sala equipada com aparelhos específicos para atendimentos de urgência e de uma ambulância, elementos que reforçam sua identidade enquanto uma instituição terapêutica capaz de prestar atendimento a pessoas com doenças graves como o câncer.

Todo atendimento oferecido no CAPC é gratuito, e a instituição é mantida por meio de doações, mensalidade de sócios efetivos, subvenções e trabalho voluntário. O centro é reconhecido como instituição de utilidade pública por sua atuação terapêutica assistencial na cidade de Florianópolis, o que demonstra seu reconhecimento social e político.

\section{RESULTADOS}

Com base nos dados coletados na pesquisa de campo e nas entrevistas, buscou-se traçar um perfil mínimo dos pacientes atendidos no CAPC, observando em que medida questões relacionadas à classe social, acesso a sistemas médicos, gravidade da doença e pertencimento religioso afetavam a escolha por esse tipo de tratamento. Os resultados mostraram uma grande heterogeneidade como característica dos pacientes dessa instituição. Na maior parte dos casos o tratamento complementar/espiritual não estava sendo utilizado em contraposição ao tratamento biomédico, mas conjuntamente a este e a outras formas de cuidado e atenção que pudessem trazer alívio e bem-estar. A busca pelo tratamento complementar/espiritual também foi apontada como uma forma de proporcionar às pessoas com câncer um espaço para a expressão dos seus sentimentos com relação à doença, o que, segundo eles, o sistema médico oficial não oferece.

Constatou-se que o CAPC recebe pacientes de várias classes sociais, pertencentes a diversas categorias profissionais: de professores universitários e empresários a agricultores e pescadores. No entanto, pode-se dizer que parte considerável de sua clientela é formada por pessoas das classes média e média-alta, que têm total acesso aos sistemas de saúde oficiais. Esse dado de certa forma reflete a realidade socioeconômica de Florianópolis, que é hoje a capital brasileira com melhor Índice de Desenvolvimento Humano (IDH) e que tem $40 \%$ da sua população pertencente às

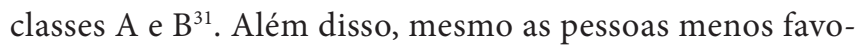
recidas economicamente que buscavam o CAPC deveriam apresentar os exames exigidos para seu tratamento. Com isso, foi possível observar que elas tinham acesso ao sistema público de saúde local, que na cidade de Florianópolis conta com um importante centro de tratamento oncológico com atendimento pelo Sistema Único de Saúde (SUS), o Centro de Pesquisas Oncológicas (CEPON).

Deste modo, pôde-se constatar que a maioria dos pacientes atendidos no CAPC possui acesso aos sistemas médicos oficiais e os utilizam. Curiosamente, duas pacientes entrevistadas afirmaram que tomaram conhecimento do CAPC por indicação de seus próprios médicos. $\mathrm{O}$ tratamento espiritual teria sido indicado como forma de "equilibrar-se emocionalmente" para suportar os tratamentos convencionais, especialmente a quimioterapia, mas não como forma de evitá-los. Na fala de uma delas:

É um arraso quando a gente recebe uma notícia dessas, parece que tudo que tu fez na vida nada mais tem valor, vai desmoronar teu mundo e tua vida acabou ali! Foi quando a minha mastologista me disse pra fazer o tratamento emocional, que ajudaria muito, lá no Ribeirão, que tinha um lugar de apoio ao paciente com câncer e eles faziam um tratamento emocional, só que era espírita. Eu sou de formação católica, mas eu não tenho nada contra que seja de outra religião, eu quero mais é me tratar [...] Só que ela deixou bem claro que lá eu ia tratar o emocional, mas que a cirurgia da mama ia ter que ser feita de todo jeito (P10, câncer de mama).

Foram encontrados ainda pacientes que tomaram decisões quanto ao tratamento alopático após iniciarem seu tratamento no CAPC, já que ali sempre é enfatizado que a pessoa com câncer não deve abandonar a medicina convencional e utilizar apenas a MAC e os tratamentos espirituais, aspecto destacado sobretudo pelos médicos que atuam na instituição:

Eu vim pra cá pensando 'vou fazer esse tratamento e vou ficar boa e dizer pro meu médico que não vou mais fazer quimioterapia, não vou fazer nada, mas eu encontrei outra ideia de cura aqui. Eu penso que fui curada quando aceitei que eu tenho sim que fazer a quimioterapia, que eu felizmente posso fazer e tanta gente não pode ou não adianta mais fazer, mas o meu câncer tá bem no início e se eu fizer tudo direitinho eu tenho mais chances de me curar totalmente (P15, melanoma).

Dentre os pacientes entrevistados, apenas dois estavam utilizando o tratamento do CAPC como alternativa ao tratamento alopático. Um deles, com um tumor no cérebro, afirmou que seus médicos não recomendaram a cirurgia devido à localização e ao tipo do tumor (em estágio avançado), e que a tentativa de tratamento quimioterápico também havia sido descartada. O tratamento do CAPC aparecia como alternativa em função de o paciente não ter indicação para formas de tratamento biomédico. 
Uma segunda paciente, com nódulo no seio, não desejava realizar tratamentos convencionais contra o câncer. No entanto, ela não tinha se submetido a uma biopsia para confirmar a malignidade do nódulo por acreditar que não faria diferença ter essa informação, pois não pretendia realizar a mastectomia nem a quimioterapia, tratamentos que ela considerava "piores que a própria doença”. Ela monitorava seu nódulo por meio de exames regulares.

Todos os demais pacientes entrevistados realizaram cirurgia e/ou tratamento convencional contra o câncer antes, durante ou após seu tratamento no CAPC.

Tem-se frequentemente a ideia de que apenas pacientes terminais ou sem possibilidade de tratamento biomédico recorrem a terapias complementares/ espirituais como a "última opção". No entanto, foram encontrados pacientes no CAPC em vários estágios da doença: recém-diagnosticados, fora de possibilidade terapêutica, reincidentes, pessoas que já haviam realizado cirurgia e tratamento convencional contra o câncer ou que estavam realizando a quimioterapia, além de pacientes que estariam com a doença sob controle por estarem há mais de cinco anos sem sintomas do câncer.

Por outro lado, havia também pacientes que estavam em tratamento no CAPC há vários anos e ainda apresentavam sintomas da doença, ou que desenvolveram metástase. Para estes, a cura era vista como um processo longo e demorado, podendo até mesmo não ocorrer em termos físicos, mas de outra ordem, como uma cura das emoções ou uma cura espiritual. Não haveria aqui uma relação puramente utilitária com o tratamento complementar/espiritual, e a lógica da adesão podia ser a mesma apresentada para o tratamento alopático:

Tem gente que me pergunta, 'ah, mas tu fez tratamento lá [CAPC] e ficou doente de novo, pra que voltar então?' Mas aí eu respondo que eu fiz tratamento no médico também, e o câncer voltou, e aí? Eu não vou voltar mais no médico porque o câncer reapareceu? Se eu já tive câncer três vezes e estou aqui falando com vocês, eu acho que é porque em todas as vezes os meus tratamentos funcionaram, funcionaram tanto que eu estou aqui, eu não morri (P4, câncer mama/tireoide).

Por fim, foi analisado o pertencimento religioso dos pacientes com câncer, pois a "crença" dos pacientes é apontada como um elemento importante que motivaria a busca e a adesão aos tratamentos espirituais, bem como a sua eficácia, em alguns $\operatorname{casos}^{32,33}$. Entre as pessoas entrevistadas, 11 se declararam espíritas; 6 espiritualistas, mas sem religião definida; 3 católicas; e 1 umbandista. No entanto, na observação cotidiana dos prontuários dos pacientes observou-se que, em sua maioria, eles se declaravam católicos, sendo espírita a segunda denominação mais frequente, seguida de sem religião ou espiritualista. Foram encontrados ainda alguns poucos registros de evangélicos, luteranos e agnósticos.

Não poderíamos afirmar, portanto, que a busca pelo tratamento espiritual seria exatamente motivada por uma crença internalizada a priori, baseada num conhecimento prévio do espiritismo, religião que está na base da instituição CAPC, ou de suas práticas terapêuticas. Na observação em campo e nas entrevistas foram observados pacientes que afirmaram nunca ter ouvido falar do CAPC antes da sua doença, e que nunca haviam utilizado quaisquer das terapias complementares/espirituais oferecidas pelo Centro.

\section{DISCUSSÃO}

No CAPC, a heterogeneidade dos sujeitos é marcada por aspectos que vão da classe social à filiação religiosa, passando pela própria doença, que para uns era quase passado, enquanto para outros era o pleno presente, colocando em questão algumas tentativas de generalização encontradas na literatura que trata das curas espirituais ${ }^{33,34}$. Nessas análises, o retrato totalizante que se busca imprimir aos pacientes lhes confere, na maioria das vezes, um lugar de passividade no qual a experiência suscitada pelo tratamento espiritual e os desdobramentos dessa terapêutica na construção do cotidiano da pessoa doente são ofuscados por ideias como as de clientelismo, "crença", conversão e submissão do paciente aos valores do grupo religioso ou às carências de ordem econômica.

No entanto, a pesquisa evidenciou que considerar exclusivamente esses fatores como motivadores da busca e adesão das pessoas com câncer a essas formas de tratamento significaria reduzir a complexidade que essa experiência representa para os sujeitos, limitando as possibilidades de sua análise. Faz-se necessário considerar a diversidade de significações envolvendo as noções de saúde, doença e cura na experiência individual dos sujeitos, pois esses conceitos não teriam aqui os mesmos significados para todos.

Neste sentido, a pesquisa mostrou que o tratamento complementar/espiritual do CAPC era avaliado pelos pacientes como uma forma válida de cuidado com diversas possibilidades de significação, tais como: potencializar o tratamento alopático, aumentar as chances de cura total da doença, tratar de outras dimensões consideradas importantes nos processos de saúde-doença (mental, emocional, espiritual), prevenir novos tumores e outras enfermidades, fortalecer-se emocional e psicologicamente, "estacionar" o câncer, compartilhar experiências e conhecimentos sobre a doença com outras pessoas, assim como ter um lugar para expressar seus sentimentos.

Certamente a expectativa de uma cura rápida e efetiva estava entre os anseios desses pacientes, mas não alcançar essa cura do 
ponto de vista biológico não determinava a adesão ou o abandono do tratamento. Foram encontrados pacientes que estavam em tratamento no CAPC há vários anos e não podiam se considerar curados do ponto de vista biológico e, ao contrário, pessoas sem sintomas da doença que permaneciam realizando tratamentos espirituais e complementares como "forma preventiva".

Para a maioria dos pacientes entrevistados, e na observação de outros pacientes durante a pesquisa de campo, as terapias complementares e a cirurgia espiritual funcionavam como uma forma de cuidado que iria além daquelas oferecidas pela biomedicina. Os pacientes de modo geral não recusavam o tratamento alopático. Ao contrário, muitos consideravam que o acesso à biomedicina era uma questão de cidadania, porém, afirmavam que ela por si só poderia ser menos eficaz dadas as limitações de sua abordagem, centrada exclusivamente no corpo:

Se não fosse o CAPC, se eu tivesse ficado só no alopático, eu não sei se eu ia ter essa força, porque tu trata o corpo, mas tem que tratar o espírito também e a cabeça para ter força, e essa parte eu tratei no CAPC (P6, câncer de mama bilateral).

Eu tinha consciência de que fisicamente eu tinha feito o que eu deveria fazer, que foi a cirurgia e os exames, eu nem precisei fazer quimioterapia porque o tumor foi detectado bem no início. Mas pelo que eu conhecia do CAPC nessa proposta que trata a pessoa de forma mais integrada, não simplesmente tratar lá o intestino, foi esse tipo de expectativa que me levou até lá, buscando algo além do tratamento físico (P3, câncer intestino).

Deste modo, observou-se que a noção biomédica da cura baseada na supressão de sintomas ou sinais de uma doença não seria o único elemento motivador para uma pessoa iniciar, continuar ou interromper seu tratamento complementar/espiritual no CAPC, mas sim o que esse tratamento poderia significar na experiência da pessoa doente ao permitir a construção de sentidos mais amplos para o câncer, trazendo alívio e bem-estar físico e psicológico.

No caso do pertencimento religioso, percebemos que as pessoas não apenas vinham de formações religiosas distintas, mas que também negociavam sentidos daquele universo terapêutico-religioso de forma a compor compreensões sobre os fenômenos de saúde-doença, o que não necessariamente resultava em uma conversão religiosa, mesmo no caso de pacientes que se tornaram voluntários do CAPC, pois alguns deles não se consideravam espíritas. Deste modo, analisou-se a adesão ao tratamento do CAPC nos termos de uma conversão terapêuti$c a^{35}$, disposição através da qual elementos religiosos/espirituais são ressaltados na compreensão dos estados de saúde-doença e formas de cuidado, sem que isso necessariamente resulte em uma adesão a determinado grupo ou denominação religiosa.

Certamente, os pacientes do CAPC compartilhavam informações acerca das cirurgias espirituais e de conceitos do espiritismo devido à popularização da doutrina espírita no Brasil, por meio da mídia e até de telenovelas. Porém, observou-se ao longo da pesquisa que a "crença" no tratamento espiritual e nas terapias complementares era algo que precisava ser criado e mantido, e não simplesmente expresso na busca por essas formas terapêuticas. Por isso, ter "fé no tratamento" não seria condição prévia para o engajamento nos tratamentos oferecidos pelo CAPC, mas sim uma ação processual através da qual o doente poderia auxiliar o organismo em sua recuperação ao elaborar ativamente sua participação no processo de cura.

\section{CONSIDERAÇÕES FINAIS}

Ao analisar a perspectiva da pessoa com câncer que busca tratamentos complementares/espirituais, esta pesquisa colocou em questão certas concepções vigentes tanto no senso comum como entre pesquisadores acadêmicos, que analisam a adesão a essas formas de tratamento com base nas ideias de "crença”, carência econômica e/ou incapacidade da biomedicina em tratar certas doenças. Observamos que as terapias complementares/espirituais oferecidas no CAPC são analisadas pelos pacientes oncológicos como formas possíveis de tratamento que podem ser acionadas de diversas maneiras durante o itinerário terapêutico, tendo por base uma lógica de cuidado diversificada e plural que não opera com as dicotomias mente/corpo, biomedicina/terapias complementares para pensar os processos de saúde e doença, nem está determinada pela gravidade da doença, condição econômica ou filiação religiosa dos sujeitos.

Não negamos que estas situações também estejam presentes como elementos motivadores da busca de pacientes oncológicos pelo tratamento complementar/espiritual. Porém, a pesquisa evidenciou que embora supridas as necessidades de ordem econômica e de acesso a sistemas médicos oficiais, e apesar da eficácia dos tratamentos oferecidos pela biomedicina, as pessoas com câncer sentem necessidade de espaços terapêuticos nos quais elas possam vivenciar e compartilhar sentimentos com relação à doença, elaborar sentidos para essa experiência que considere seu universo sociorrelacional e participar mais ativamente das decisões quanto ao seu tratamento.

\section{AGRADECIMENTOS}

Agradecemos à CAPES e ao CNPQ pelo apoio financeiro que viabilizou a realização desta pesquisa e ao professor Marcelo Mercante, pelo auxílio com a tradução do resumo. 


\section{REFERÊNCIAS}

1. Organização Mundial da Saúde. Traditional Medicine Strategy 2002-2005. Geneva; 2002

2. BRASIL. Ministério da Saúde. Secretaria de Atenção à Saúde. Departamento de Atenção Básica. Política Nacional de Práticas Integrativas e Complementares no SUS - PNPIC-SUS. Brasília; 2006.

3. Spadacio C, Barros NF. Uso de medicinas alternativas e complementares por pacientes com câncer: revisão sistemática. Rev Saúde Pública. 2008;42(1):158-64.

4. Luz MT. Cultura contemporânea e medicinas alternativas: novos paradigmas em saúde no fim do século XX. PHYSIS: Rev Saúde Coletiva. 2005; 15(Suppl):145-76 .

5. Barros NF. A construção da medicina integrativa: um desafio para o campo da saúde. São Paulo: Hucitec; 2008.

6. Langdon EJ. Breve histórico da antropologia da saúde. In: A negociação do oculto: Xamanismo, família e medicina entre os Siona no contexto pluri-étnico. Trabalho apresentado para o Concurso de Professor Titular na Universidade Fedral de Santa Catarina; 1994.

7. Montero P. Da doença à desordem: a magia na umbanda. Rio de Janeiro: Graal; 1985.

8. Giumbelli E. Espiritismo e medicina: introjeção, subversão, complementaridade. In: Isaia AC, editor. Orixás e espíritos: o debate interdisciplinar na pesquisa contemporânea. Uberlândia: EDUFU; 2006. p. 283-304.

9. Rabelo MCM. Religião e cura: algumas reflexões sobre a experiência religiosa das classes populares urbanas. Cad Saúde Pública. 1993;9(3):316-25.

10. Rabelo MCM. A construção do sentido nos tratamentos religiosos. RECIIS. 2010;4(3):3-11

11. Rabelo MCM, Motta SR, Nunes JR. Comparando experiências de aflição e tratamento no candomblé, pentecostalismo e espiritismo. Religião e Sociedade. 2002;22(1):93-121.

12. Maues RH. Cura e religião: pajés, carismáticos e médicos. In: Fleischer SR, Tornquist CS, Medeiros BTF, editors. Saber cuidar, saber contar: ensaios de antropologia e saúde popular. Florianópolis: Ed. da UDESC; 2010. p. 125-42

13. Santos RV, Coimbra Jr. CEA, editors. Saúde e povos indígenas. Rio de Janeiro: FIOCRUZ; 1994

14. Langdon EJ, Granelo L, editors. Saúde dos povos indígenas: reflexões sobre antropologia participativa. Rio de Janeiro: Contra Capa Livraria/ ABA; 2004.

15. Maluf SW. Mitos coletivos e narrativas pessoais: cura ritual, trabalho terapêutico e emergência do sujeito nas culturas da Nova Era. Mana. 2005;11(2):499-528.

16. Tavares FRG. Ascensão e profissionalização da terapêutica alternativa no Rio de Janeiro (anos 80-90). PHYSIS: Rev Saúde Coletiva. 1999;9(2):75-98.

17. Tavares FRG. A diversidade da rede terapêutica alternativa no Rio de Janeiro. PHYSIS: Rev Saúde Coletiva. 2002;12(2):325-44.
18. Tavares FRG. Legitimidade terapêutica no Brasil contemporâneo: as terapias alternativas no âmbito do saber psicológico. PHYSIS: Rev Saúde Coletiva. 2003;13(2):83-104

19. Terzioglu A. Experiencing and explaining cancer: a critical study of turkish modernity through the cancer patients' illness narratives [thesis]. New York (NY): The City University of New York. Graduate Faculty in Anthropology; 1999.

20. Luxardo N. Las "alternativas" del cáncer: configuración y dinámicas de un campo de atención no formal [tese]. Buenos Aires: Universidad de Buenos Aires. Facultad de Ciencias Sociales; 2007.

21. Anthropologie des soins non conventionnels du cancer [internet]. Anthropologie \& Santé. Revue Internationale Francophone d'Anthopologie de la Santé. 2011.[cited 2011 June 5]. Available from: http://anthropologiesante.revues.org/147

22. Velho G. Individualismo e cultura. Rio de Janeiro: Jorge Zahar; 1987.

23. Good B. Medicine, rationality, and experience. Cambridge: Cambridge University Press; 1994

24. Rabelo MCM, Alves PC, Souza IM. Experiência de doença e narrativa. Rio de Janeiro: FIOCRUZ; 1999

25. Mattingly C. Healing dramas and clinical plots: The narrative structure of experience. Cambridge: Cambridge University Press; 1998.

26. Garro LC, Mattingly C, editors. Narrative and the cultural construction of illness and healing. Berkeley: University of California Press; 2000.

27. Bauman R, Briggs C. Poética e performance como perspectivas críticas sobre a linguagem e a vida social. Ilha - Rev de Antropologia. 2006;8(1/2):185-229.

28. Langdon EJ. Performance e sua diversidade como paradigma analítico: a contribuição da abordagem de Bauman e Briggs. Ilha - Rev de Antropologia. 2006;8(1/2):162-83.

29. Laderman C, Roseman M, editors. The performance of healing. New York: Routledge, 1996

30. Conselho Nacional de Saúde. Resolução 196/96. Diretrizes e Normas regulamentadoras de pesquisa envolvendo seres humanos. Brasília: Ministério da Saúde; 1996.

31. Qualidade de Vida. 2011. [cited 2011 April 19]. Available from: http:// santacatarinabrasil.com.br/pt/qualidade-de-vida/

32. Levi-Strauss C. Antropologia estrutural. São Paulo: Cosac Naify; 2008.

33. Greenfield S. Cirurgias do além: pesquisas antropológicas sobre curas espirituais. Petrópolis: Vozes; 1999.

34. Camargo CPF de. Kardecismo e umbanda. São Paulo: Pioneira; 1961.

35. Aureliano WA. Espiritualidade, saúde e as artes da cura no contemporâneo: indefinição de margens e busca de fronteiras em um centro terapêutico espírita no sul do Brasil [tese]. Florianópolis (SC): Universidade Federal de Santa Catarina. Programa de Pós-Graduação em Antropologia Social; 2011

Recebido em: 05/04/2012 Aprovado em: 19/09/2012 